\title{
When it Hurts: Too Young or Too Old
}

\section{Penny Gilbert ${ }^{*}$}

Baptist Health System, Case Management, 1740 Nicholasville Road, Case Management Department, Lexington, KY 40503, United States

*Corresponding author: Penny Gilbert, Baptist Health System, Case Management, 1740 Nicholasville Road, Case Management Department, Lexington, KY 40503, United States, Tel: 6067760208; E-mail: penny.gilbert@BHSI.COM; pennygilbert174@hotmail.com

Received date: December 26, 2014, Accepted date: January 16, 2015, Published date: January 19, 2015

Copyright: $\odot 2015$ Gilbert P. This is an open-access article distributed under the terms of the Creative Commons Attribution License, which permits unrestricted use, distribution, and reproduction in any medium, provided the original author and source are credited.

\begin{abstract}
:
Background: Child and elder abuse and/or neglect have been a long standing healthcare topic. Family structure, socioeconomics, and developmental tasks often increase the burden for healthcare providers to identify abuse and neglect of the very young or elderly. These same factors often increase the risks for abuse or neglect. However, there are individual state laws requiring healthcare professionals report suspected abuse/neglect, else face legal ramifications. Abuse may appear in multiple forms according to Dong (2012). These include maltreatment in the form of psychological, physical, and sexual abuse, neglect and financial exploitation. Childhood abuse often leads to psychological issues that follow the individual into adulthood. It is important nurses learn to recognize signs and symptoms of abuse and/or neglect and report per local regulation requirements. Advocating for one's patient(s) and for one's self creates positive outcomes for all involved.
\end{abstract}

Purpose: The purpose of this manuscript is to encourage nurses across the United States to acquire education on abuse and/or neglect reporting requirements and to take action when appropriate, advocating for those that cannot self-advocate.

Conclusions: It is imperative nurses recognize the signs and symptoms of abuse and neglect, advocate for those that cannot do so and follow local state regulations/laws. Literature research reveals approximately 120 children are substantiated as experiencing abuse and/or neglect per hour. In the U.S. greater than 3.4 million children will live with a grandparent due to mother substance abuse disorder and the number of abused or neglected elderly is simply unknown.

\section{Introduction}

The topic of abuse and neglect are, in general, difficult subjects to discuss. In healthcare it is, unfortunately, a common topic encountered or identified within all settings of healthcare. Developmental task of families throughout the lifespan can often be a challenge and appears to set the environment for potential or real abuse and neglect. Family structure is a factor in the face of abuse and neglect, for children and the elderly. In the United States our definition of family is rapidly changing and it is not uncommon to find grandparents raising grandchildren or adult children caring for their elderly parents. Not reporting suspected abuse or neglect can lead to real and serious outcomes for the healthcare provider. "In the United States failure to report by a mandated reporter (including nurses) can result in fines ( $\$ 100$ to $\$ 500$ ) and prison time (10 days to 5 years)" [1].This paper will further explore abuse and neglect with the importance of family structure, characteristics of a healthy family, and collaborative interventions for health promotion.

\section{Family Structure and Characteristics}

Family structure describes the members and relationships among several or many, depending upon the culture. "The meaning of family among the Hmong of northern Laos may include hundreds...in Germany and Japan families are small" [2]. Therefore, family structure is all inclusive of the members, relationships among the members, and demographics of each member. Cultural competence is important when identifying true abuse/neglect situations. The National Center on Elder Abuse defines it as "intentional or neglectful acts by a caregiver or trusted individual that lead to or may lead to harm" [3]. Healthy family characteristics include, "healthy interaction among members, enhancement of individual development, effective structuring of relationships, active coping effort, healthy environment and lifestyle, and regular links with the broader community" [2]. However, nurses must recognize traditional family is becoming rare. "Due to the increasing prevalence of this community health dilemma (family member abuse/neglect) there is an immediate need for counseling, support groups, and social service navigation" [4]. It is important for resources, education and support be provided to family members.

\section{Developmental Tasks \& Family Diversities}

Developmental tasks of families throughout the lifespan can often challenge healthcare providers. "Developing new family treatments and adapting interventions to the particular needs of families is a challenge for the field". Developmental level describes the study of the family, according to Allender et al. [2] from a life-cycle perspective. Duval [5], describes eight cycles within family development: (1) Family beginning; (2) birth of first child; (3) pre-school; (4) school; (5) adolescence; (6) child leaves home; (7) post parental stage; and (8) retirement. Family functions and roles are an important developmental assessment.

The diversity of family within the U.S. ranges from single parents to grandparents acting as the primary caregiver for grandchildren and 
Page 2 of 3

adult children caring for elderly parents, just to name a few. "In the US more than 3.4 million children live with a mother who has a substance abuse disorder (SUD) and at some time in their life will be cared for by a grandmother" [4]. When responsibilities change from that of the traditional family, whether through kinship or obligation, there are often gaps and stressors that affect all involved. "Role ambiguity and conflict are issues of concern" [4].

\section{The Numbers}

Data collection categories for family developmental assessment are well described by Allender et al. [2] as family strengths and self-care capabilities, family stresses and problems and family resources. Family roles identify several elements but one of the most important ones is that of authority and power when evaluating a situation of child or elder abuse or neglect. "Understanding the association among risk factors and outcomes commonly used in research on family caregivers can inform the assessment and interventions" (Figure 1) [6].

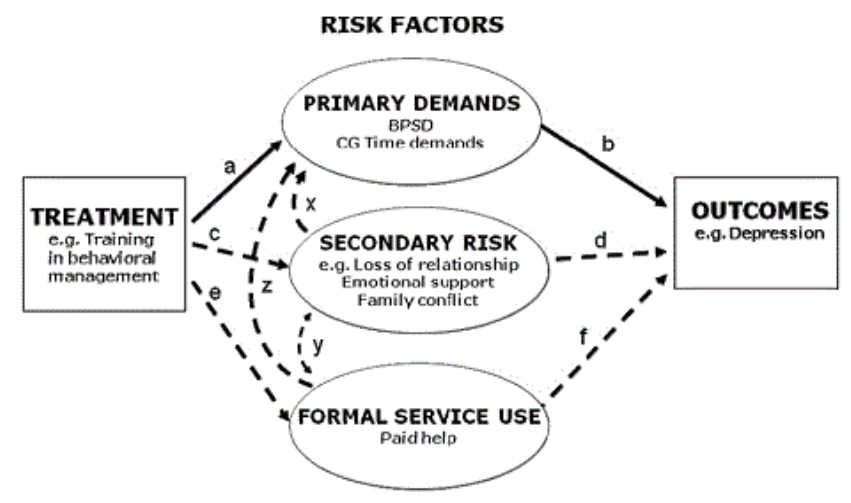

Figure 1: Stress process model showing the effect of treatment on risk factors and outcomes.

Note: Solid paths indicate the effects of a single treatment design. The dotted paths indicate the multiple influences and reciprocal effects between constructs [6].

According to Nguyen [7], one child is reported with maltreatment every 5 seconds, while substantiated every 30 seconds. That equates into 12 children reported per minute with 120 cases substantiated per hour. Richardson further identifies safeguarding vulnerable people as a professional responsibility, especially when opportunities to protect are missed by others. "Safeguarding is the protection of vulnerable groups from abuse and/or neglect". Each of us has a responsibility, whether professionally or ethically to safeguard anyone deemed vulnerable. All states including the District of Columbia and American Samoa, Guam, the Northern Mariana Islands, Puerto Rico and the Virgin Islands have designated professions who are mandated by law to report maltreatment such as abuse and or neglect, according to Mandatory Reporters. These individuals include social workers, teachers, principals and other school personnel, physicians, nurses, and other healthcare workers, child care providers, medical examiners/ coroners and law enforcement officers.

\section{Collaboration, Interventions and Health Promotion}

Societal problems continue to exist just as they have in the past. Reported cases of abuse and neglect in all ages, but especially in ages of greater risk, young and elderly are continuously reported. We also see those with less means or in true poverty, lower ability to obtain medical and mental health services, thus true disparities, which may contribute to abuse and neglect. These disparities range from socioeconomic to racial and ethnic status. "Health literature has long acknowledged inequities in health care for minorities". Casual thinking continues to emerge as does the necessity for changes in education of nurses, patients and communities.

Community and population health have recently risen to the forefront within the healthcare arena and with it greater burden on community health nurses. It is essential all nurses recognize the responsibility and standards associated with reporting of abuse and neglect along with the connection of such events to population health. Reporting regulations may be stringent, therefore there is importance in recognizing multiple states identify specific timeframes in which the suspected abuse/neglect must be reported. Nurses should know their individual state law requirements and abide by such laws.

Often nurses do not think of abuse or neglect for elderly when in a long-term care facility, but incident reporting is often high for such environments. The National Center on Elder Abuse Administration on Aging (NCEAAA) recognizes that today we simply do not know the number of elderly suffering from abuse or neglect. However, it is noted that females are abused more than males and the older an individual is, the more likely he/she will be abused (NCEAAA).

"The sharing of data, particularly health data, has been an important tool for the public health community...child maltreatment is a serious public health issue that could be better mitigated with interoperability" [7]. Hoover and Polson provide an excellent guide that may be useful in assessing patients for abuse. The more we learn, share, and educate others, in other words collaborate, the closer we come to solutions. Collaboration of caregivers in the fight against abuse and neglect for any population may be the foundation for solving or at least reducing this horrible healthcare dilemma.

\section{Summary}

In summary, evaluation of the family is an essential duty of the nurse. Family structure, development and foundations vary, thereby creating unique and challenging interventions. It is imperative that the nurse recognize and report suspected acts of abuse or neglect, especially in children and the elderly. The nurse should be acutely aware of individual state reporting requirements in efforts to stay compliant and prevent legal actions which may occur with noncompliance. Knowing the risk factors that lead to abuse and/or neglect, using appropriate screening tools to guide in its identification, are key steps in identifying and reporting such events. Collaboration and communication are additional key factors in reducing such events while creating safety for these vulnerable populations.

\section{References}

1. DeMattei RR, Sherry JS (2011) Initiating discourse on recognizing and reporting child abuse. Canadian Journal of Dental Hygiene, 45: 253-258.

2. Allender JA, Rector C, Warner KD (2010) Community health nursing: Promoting \& protecting the public's health (7th ed.). Philadelphia, PA: Lippincott, Williams \& Wilkins. 
3. Hoover RM, Polson M (2014) Detecting elder abuse and neglect: Assessment and intervention. American Family Physician Journal, 89: 453-460.

4. Lange B, Greif S (2011) An emic view of caring for self: Grandmothers who care for children of mothers with substance use disorders. Contemporary Nurse: A Journal for the Australian Nursing Profession, 40: 15-26.

5. Duvall EM (1948) Human Development Program. Paper presented at the National Conference on Family Life, Washington, DC.
6. Zarit S, Femia E, Kim K,Whitlatch C (2010) The structure of risk factors and outcomes for family caregivers: Implications for assessment and treatment. Aging \& Mental Health, 14(2), 220-231.

7. Nguyen LH (2014) A public health response to data interoperability to prevent child maltreatment. American Journal of Public Health, 104: 2043-2048. 